\title{
The Smart Attendance Monitoring System
}

\author{
M. Sasi Chandra, Radhika Baskar
}

\begin{abstract}
As an important part of class teaching, attendance plays a key role in the evaluation of teaching. At the beginning and end of class, it is usually checked by the teacher, but it may appear that miss someone or some students answer multiple times. To overcome this issue, the latest technology is used to detect the faces and recognize the faces using python programming. Face Detection is the bestsignificant topics of computer technology in machine learning application. This technology has been available for some years now and is being used all over the world. In this paper, capturing the images of students from the database and faces are detected by the algorithm and then it is acknowledging with the database and at last, the attendance is recorded. For detecting faces Viola-Jones facial identification algorithm is used and for recognizing the faces from the databases( $\mathrm{LBPH})$ Local Binary Patterns Histograms technique is used.
\end{abstract}

Keywords: Python, Viola-Jones algorithm, Local Binary Patterns Histograms (LBPH), machine learning.

\section{INTRODUCTION}

The most difficult task in various schools/colleges is student attendance. Every schools/college are taking attendance by using an attendances sheet or biometric. But using systems consume so more time. Majorly student appearance sheet which is given to the faculty members is used to take the student attendance, but it may appear that miss someone or some students answer multiple times. In other cases, the appearance sheet may be misplaced or gone. To reduce such problems a smart attendance management system be needed.

Lately's video-based face identification has experience with immediate consideration and it will be an important topic to researchers for the people's identification in the field of image handling for public'sproof of identity. The main focus for the researchers is face identification, more probably video-based face identification because videos observation, attendance checking in different organization and parallelsafety purposes are based on it. This paper indicates that how to facialidentification techniques be effective in the educational field for an effectual attendance scheme will repeatedly record the existence of aregistered individual within their own class. The face acknowledgment

Revised Manuscript Received on September 10, 2019.

M. Sasi Chandra, UG Scholar, Department of Electronics and Communication Engineering, Saveetha school of engineering, Saveetha Institute of Medical and Technical Sciences, Chennai, Tamilnadu, India (Email: sasichandra.morukurthi@gmail.com)

Dr. Radhika Baskar, Associate Professor, Department of Electronics and Communication Engineering, Saveetha school ofengineering, Saveetha Institute of Medical and Technical Sciences, Chennai, Tamilnadu, India.

(Email: radhikabaskar@saveetha.com) framework includes different advances however identification and acknowledgment of the face are generally significant. Initially, the camera is utilized to record the video of the whole class for a couple of moments after that framework read the settings from the video and imprint the appearances in the study hall. In the wake of catching the edges, the framework plays out the face location which isolates the face and non-face objects. For face location, Viola Jones calculation which comprises of an AdaBoost calculation and haar-like element classifiers is utilized for face discovery. Viola-Jones is a reasonable calculation is utilized for edited appearances. The identified trimmed appearances are handled further for face acknowledgment utilizing a reasonable calculation. Removed highlights of the picture which contain the attributes of the nearness of register individual inside their classes. Face database which is our preparation set will perceive by contrasting the information picture of a specific individual. Participation is enlisted in an exceed expectations sheet subsequent to finding the legitimate match. In this paper, for every individual, 30 pictures with various postures will comprise of this paper. We get distinctive test results by shifting the size of the preparation set.

\section{LITERATURE SURVEY}

In the literature inspection of videos and picture-based face identification, we conclude that various face recognition techniques and observed facial detection and facialidentification are involved. Detection plays a major role to get a high recognition rate, in recent years' number offacial detection and facialidentificationalgorithms are developed by researchers to Implement the classroom attendance system a huge amount of work should be done attendance system [1-6]. In [1] There are two types of automatic address based systems. In [7] \& [8] For face detection, there are different views. [7] To get a better system for face recognition most of the student's proposed different steps like PCA and LDA.

\section{PROPOSED METHODOLOGY}

\section{A. Structuralplan:}

The construction of the smart appearance basic scheme for that a high megapixel camera which can cover the whole class as shown in Fig [1]. The project is portable and easy to use. To start our plan first we need a database which already filled with student images. The images are crop to the size

Published By: 
of $24 \times 24$. Now as soon the camera will turn on a few seconds and it will record the whole class and it will stop automatically. The system will take one of the frames of the video and it will capture who are sitting in the classroom the algorithm will detect the faces and then it will crop into 24 X 24 size. After the process, the cropped faces are sent to the recognizer. The database will compare the faces and it will recognize and mark the attendance

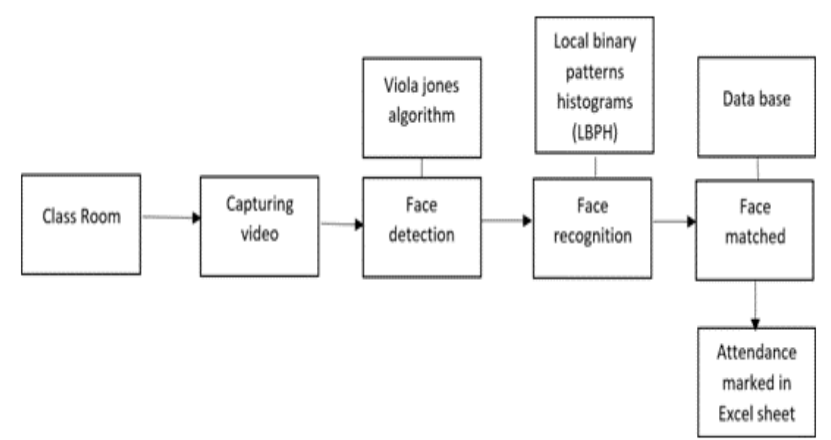

Figure 1. Block diagram of smart attendance monitoring system

\section{B. Explanation on Face detection and} Facerecognition:

Face detection which is the trending topic in the computer science field in machine learning application. This technology is available for some years now and is being used all over the world

The main methodologies for facial detection

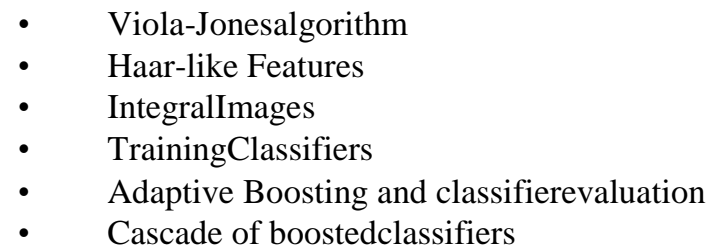

\section{Viola-Jones algorithm:}

This is the algorithm that lies at the foundation of the open CV library and this is one of the most powerful algorithms for computer vision. Training and detection at two stages in viola Jones algorithm.
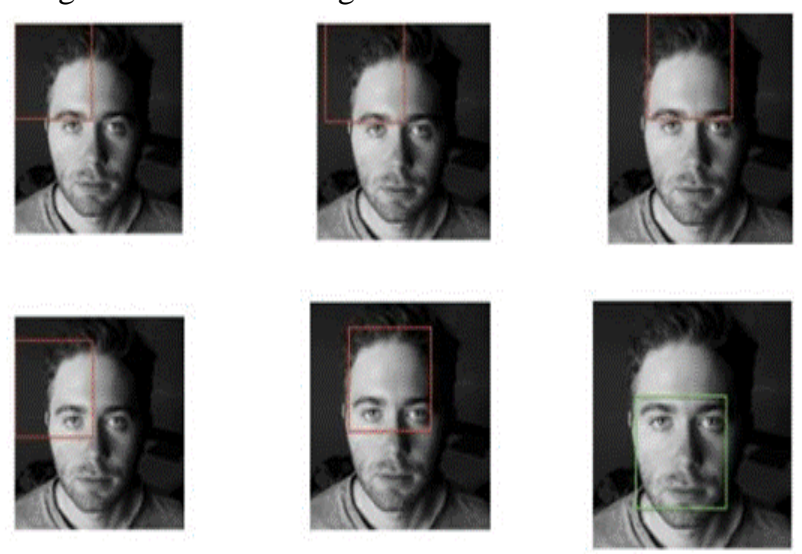

Figure 2: Working of Viola-Jones algorithm

In detection, the first step is to convert an image into a grey scale. After that, the algorithm will search for a face in the image from the left-top corner and checks as it moves side step by step towards the right. the algorithm checks the human facial characteristics like cheeks, forehead, eyebrows, eyes, etc., if all the characteristics are detected, then it is considered as a face.

\section{Haar-like Features:}

Haar-like characteristics are computerized image characteristics using facial detection. They are three types of features mainly used. They are Edge, line, four rectangle features.

When the Haar-like features are applied to the grayscale image, it will detect the dark shade in the image and the four Haar-like features are applied to specific parts of the image. After features are applied to the image, those specific parts are converted into pixels depending upon the grayscale values (0-255). It will do normalization for optimizing, assign values to the pixels from the range of 0 to 1 with 0 indicating black and 1 indicates white. it will add up the pixel values in each feature and it will differentiate their sums. the difference is used to identify the features of the image

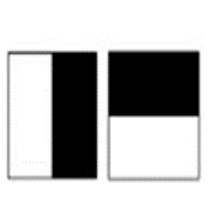

Edge Features

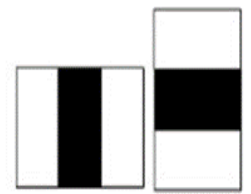

Line Features

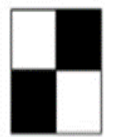

Four-rectangle Features
Figure 3.1: Haar-like features

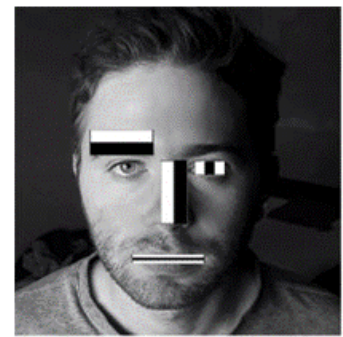

Figure 3.2: Haar-like Features are applied to the image
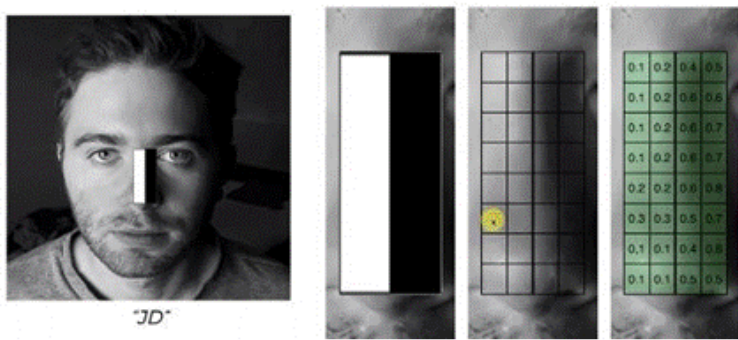

Figure 3.2: Converting features into pixel intensity

\section{Integral image:}

To perform calculations, In rectangle box, we need to add up all the values. This may be quite a costly exercise in terms of computations since the higher quantum of time is required to add elements in the rectangle so there is an easy method to reduce the time required for computation -

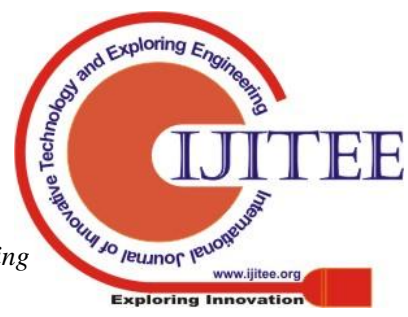


Integral imaging technique. The first step of this technique is to take the image in the aspect of pixels and to assign the values randomly to the pixels from 0 to 10 . For each specific pixel, an integral image is calculated by adding all the elements bound by the area from the top left corner to its actual position in the pixel. After that, it is easy to calculate the specific feature values by the integral image.
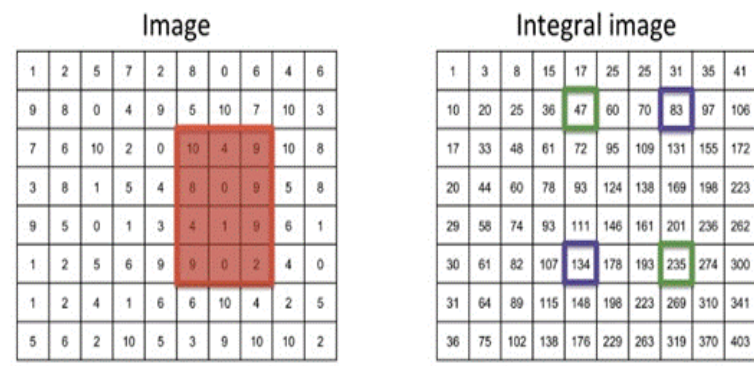

$235-83+47-134=65$

Figure 4: Integral image

\section{Training classifier:}

Fortraining,theimagesarecroppedinto24X24pixels.Itwillex tractthefeaturesfromtheimages, it will check the threshold whether the image contains facial features or not. This can be done by training the thousands of facial and non-facial images. The algorithm will check the feature that contains in the training set and conclude that image has the facial features ornot.

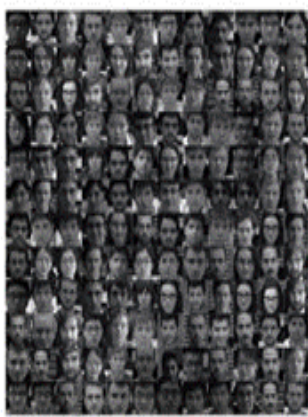

Face Images

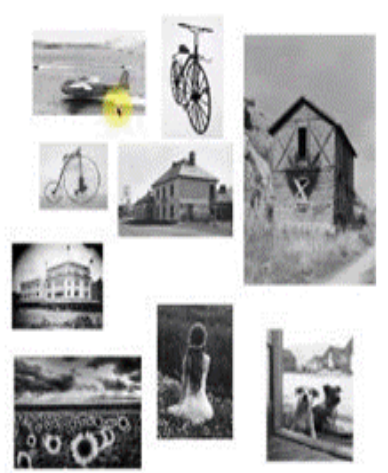

Non-Face Images
Figure 5: Training Facial and non-facial images

\section{Adaptive Boosting and classifierevaluation:}

This algorithm takes one image of 24 by 24 pixel. The possible outcome features of one image are a hundred and eighty thousand. There will be a huge amount of data is extracted from the image.it is practically impossible to do in real time. So that adaptive boosting is used for making the classifier strong and reduce the time computation. The classifier will do summation of the features and their weights of the images. The classifier will take important features of face and stored in the classifier that makes the classifierstronger.

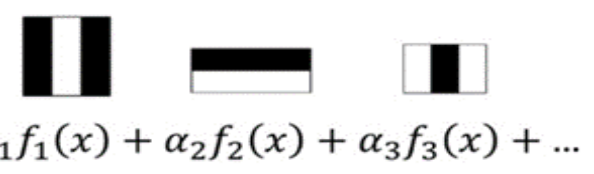

$$
F(x)=\alpha_{1} f_{1}(x)+\alpha_{2} f_{2}(x)+\alpha_{3} f_{3}(x)+\ldots
$$

Figure 6: Formula for adaptive boosting classifier

Cascade of boosted classifiers:

In real time for detecting the faces will take some time. To overcome the problem, we will go with cascade method the main step of method is to check the features from the input image from classifier ifitisavailableit willcheckthenext feature.Ifnot,it willberejected.Theclassifierwill check the important features from the face it will take milliseconds to detect thefaces.

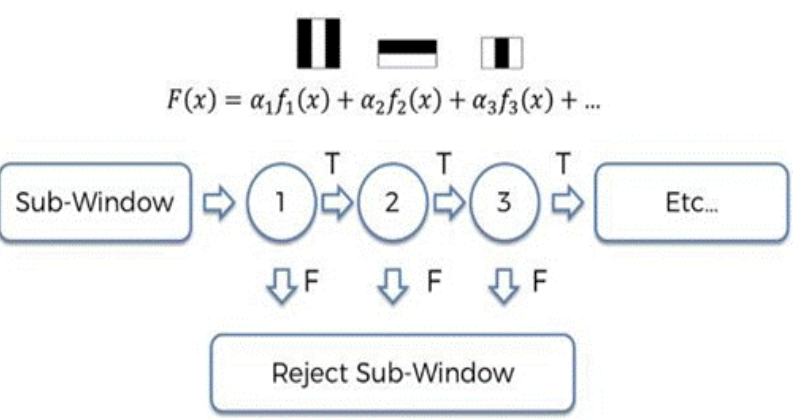

Figure 7: Cascade of boosted classifier

\section{Face recognition:}

OpenCV likewise has worked in highlights like LBPH neighborhood parallel examples histograms. In your life, we won't get careful lightning condition for both Eigen faces calculation and fisher faces calculation. LBPH recognizer is an advancement to show signs of improvement results. LBPH manages to contrast the pixels and another pixel which are in the picture.

The LBPH Face Recognizer Process the $3 \times 3$ window and moves it crosswise over one picture. The working of LBPH face recognizer is to contrast the pixels and different pixels. it will take check the neighbor pixel's qualities neither not exactly or equivalent to 1 remaining will be 0 . It will take $0 / 1$ qualities in $3 \times 3$ window it will take clockwise way. The particular request like 11100011 which demonstrates the specific territory in the picture. After complete doing this in the picture, it has a rundown of nearby double examples.

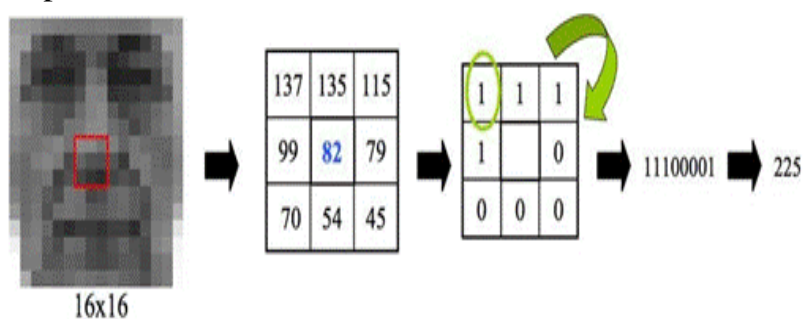

Figure 8: LBPH Face Recognizer Process

A After Subsequent to getting the rundown of neighborhood parallel examples, by utilizing the paired to decimal change it can shape a histogram consequently into 
decimal qualities. At last for each attempt and picture has one histogram for the future reference utilized for utilized by the recognizer. The calculation tracks every histogram has a place with a distinct individual. Just the face recognizer pursues the means: from the video catch, One Frame is sent to the face is measure will deliver a histogram from that picture it will separate with the histogram picture and the database picture. Finally, it finds the more noteworthy precision and gives the yield as an ideal match with the individual name.

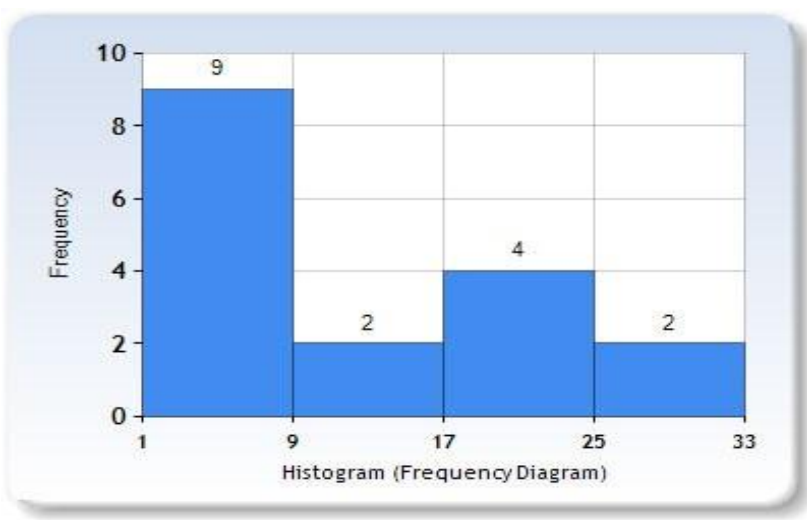

Figure 9: Histogram image

\section{METHODOLOGY}

In this project, the scheme has to monitor some key methods should be followed as written:

- Developing a database by capturingfaces

- $\quad$ Real-time videocapturing

- Facial detection

- Facial recognition

- Markingattendance

Developing a database by capturing faces:

The students must enroll their images with their names to the database. Student's faces are a crop in a specific region of a face. In this paper, the algorithm will take 30 images of the student in a few seconds and store in a database and here, the number of schoolchildren is 5. thus, generally 150 images are prepared.

\section{Real-time video capturing:}

As we discussed, we must have a generally excellent quality megapixel camera for better discovery and distinguishing proof the camera recorded a film for a 5 seconds. it will be overseen further for facial recognition.

\section{Facial detection:}

In the wake of getting the video, the method will take one of the frames and reads it after that it will stop automatically. In this paper to scan the faces open-source open cv library which is in the python is used for facial detection based on a Viola-Jones algorithm.

\section{Face identification:}

This is the main module for the project it will differentiate between the trained pictures and the tested pictures to produce a face recognition system. To execute the recognition operation LBPH algorithm is mostly used in the module.

\section{Registering attendance:}

After finishing the previous module that recognizes faces are marked as present in the attendance sheet. On the off chance that the distinguished face has been perceived, at that point it denotes the participation in the exceeds expectations sheet. To send out information to exceed expectations pandas library is utilized.

\section{RESULT AND DISCUSSION:}

The output of the project is shown below: Fig.10.1 shows how The algorithm gives the result when the given image is perfectly matched with the database image. In this project we are taking 10 student images, 30 students for each person for better accuracy in the classroom to perform the experiment, the attendance is marked in the excel sheet automatically Fig.10.2

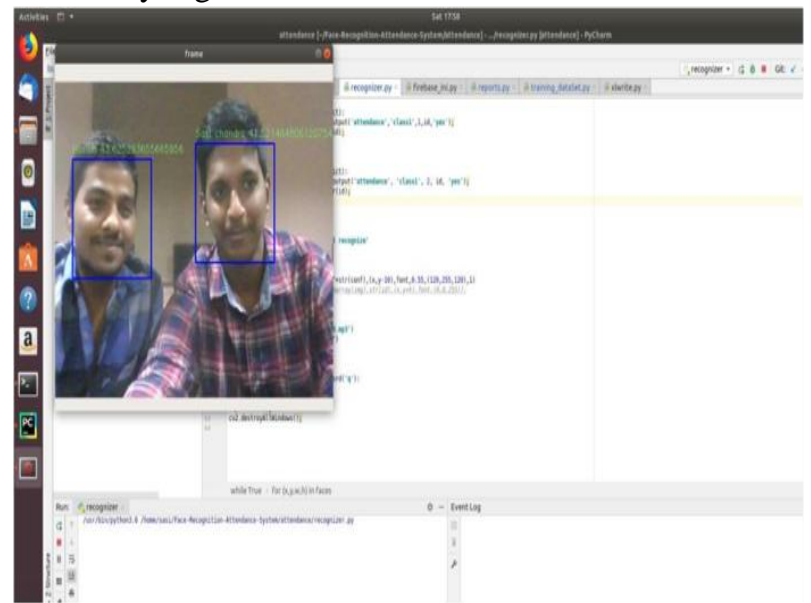

Figure 10.1: shows the output of this experiment.

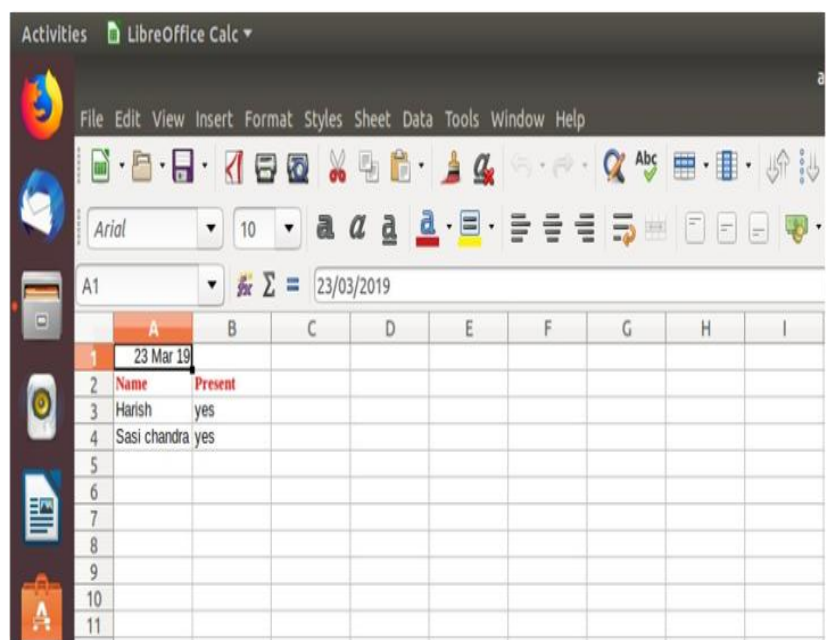

Figure 10.2: Attendance marked in the excel sheet

\section{CONCLUSION}

This project on Facial detection and facial detection has given an opportunity to study in the field of face recognition. In this project, $\mathrm{LBPH}$ is used for Characteristics extraction and recognizer. The observation says Local Binary Patterns Histograms (LBPH) In some suitable conditions it will give better performance with better output. 


\section{REFERENCES}

1. AjinkyaPatil and Mrudang Shukla, "Implementation of Classroom Attendance System Based on Face Recognition in Class", IJAET, Vol. 7, Issue 3, pp. 974979, July2014.

2. NirmalyaKar, MrinalKantiDebbarma, AshimSaha, and DwijenRudra Pal, "Study of Automated Attendance System using Face Recognition Technique", International Journal of Computer and Communication Engineering, Vol. 1, No.2, pp.100-103, July2012.

3. Rekha AL and Dr. Chethan H K, "Automated attendance system using face recognition throughvideosurveillance",InternationalJournalforTechno logicalResearchinEngineeringVol. 1, Issue 11, pp.13271330,July-2014.

4. Naveed Khan Baloch, M. Haroon Yousaf, Wagar Ahmad, M. Iran Baig, "Algorithm for EfficientAttendanceManagement:FaceRecognitionbased Approach",IJCSI,Vol.9,Issue4,No 1, pp.146-150, July2012.

5. Priyanka Wagh, RoshaniThakare, JagrutiChaudhri, Shweta Patil, "Attendance System Based on Face Recognition using Eigen face and PCA Algorithms", IEEE International Conference on Green Computing and Internet of Things, pp. 303-308,2015.

6. CAO Wei, Feng Bin, "Attendance System Applied in Classroom Based on Face Image", International Symposium on Computers \& Informatics, pp.1631-1638, Mar2015.

7. MayankChauhan and MukeshSakle, "Study \& Analysis of Different Face Detection Techniques", (IJCSIT) International Journal of Computer Science and InformationTechnologies, Vol. 5 (2), pp.16151618,2014

8. Ms. VarshaGuptaandMr.DipeshSharma, "AStudyofVariousFaceDetectionMethods" International Journal of Advanced Research in Computer and Communication Engineering Vol. 3, Issue 5, pp. 6694 6697, May2014.

9. M. Patil, Dr. Satish R. Kolhe, Dr. Pradeep M. Patil, "Face Recognition by PCA Technique", Second International Conference on Emerging Trends in Engineering Technology, pp.192-194, Dec 2009.

10. AjitK. Ushir, AjinkyaA. Shete, Sanika N. Tiwari, Vasanti R. Vishwakarma, Mahesh Sanghavi, "Mathematical Modeling for Face Recognition", International Conference on Recent Trends in Engineering \& Technology ICRTET(3), pp.13-16, May2013

11. SaurabhP.Bahurupi, D.S.Chaudhari, "Principal Component Analysis for Face Recognition" InternationalJournalofEngineeringandAdvancedTechnolo gy(IJEAT)ISSN:2249-8958,Vol. 1, Issue-5, pp.91-94, June2012.

12. Liton Chandra Paul, Abdulla Al Sumam, "Face Recognition Using Principal Component Analysis Method", International Journal of Advanced Research in Computer Engineering \& Technology (IJARCET) Vol. 1, Issue 9, pp.135-139, Nov2012

13. Parvinder S. Sandhu, Iqbaldeep Kaur, Amit Verma, Samriti Jindal, Inderpreet Kaur, ShilpiKumari, "Face Recognition Using Eigenface Coefficient and Principal Component Analysis", International Journal of Computer, Electrical, Automation, Control and Information Engineering Vol. 3, No. 4, pp.10361043,2009 .

14. KeLu, Zhengming Ding, Jidong Zhao, and Yue Wu, "Video-based Face Recognition", 3rd International Congress on Image and Signal Processing, vol.1, pp.232235,2010.

15. Aruni Singh, Sanjay Kumar Singh, ShrikantTiwari, "Comparison of Face Recognition
AlgorithmsonDummyFaces", TheInternationalJournalof Multimedia\&ItsApplications,Vol.4, No.4, pp.121-135, Aug2012.

16. PallabiParveen, BhavaniThuraisingham, “ Face Recognition using Various Classifiers: Artificial Neural Network, Linear Discriminant and Principle Component Analysis", Proceedings ofthe18thIEEEInternationalConferenceonToolswithArtifi cialIntelligence,pp.179-186,2006. 